> L'administration de cellules souches adultes est une option thérapeutique d'intérêt pour les patients présentant une artériopathie des membres inférieurs ou un infarctus du myocarde. L'efficacité de l'injection de différents types de cellules souches/progénitrices adultes autologues dérivées de la moelle osseuse, du sang périphérique ou encore de tissus a été évaluée dans des modèles expérimentaux. Ces cellules, notamment celles d'origine médullaire ou adipocytaire, font l'objet d'essais cliniques. Cette revue synthétise les principaux mécanismes moléculaires et cellulaires sous-tendant l'action des cellules souches/progénitrices adultes sur le compartiment vasculaire et propose quelques approches pour améliorer leur efficacité thérapeutique chez les patients ischémiques. <

\section{Thérapies cellulaires pro- angiogéniques dans le traitement des pathologies ischémiques}

Jean-Sébastien Silvestre

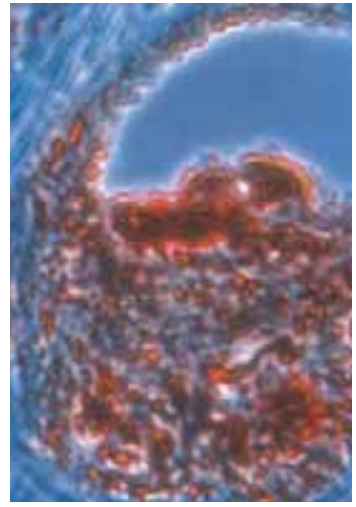

Paris-Cardiovascular Research Center-Inserm U970, Hôpital européen Georges Pompidou, 56 , rue Leblanc, 75015 Paris, France. jean-sebastien.silvestre@ inserm.fr
Les pathologies ischémiques restent une cause importante de morbidité et de mortalité dans les pays industrialisés malgré le développement de nouvelles modalités thérapeutiques comme les approches pharmacologiques ou chirurgicales. Ainsi, l'artériopathie des membres inférieurs est toujours associée à une morbidité élevée et à une baisse de la qualité de vie alors que l'ischémie myocardique, à l'origine d'une insuffisance cardiaque post-infarctus, est source d'une mortalité élevée. À partir des données expérimentales démontrant l'efficacité de l'injection de cellules souches ou progénitrices sur la fonction cardiaque après un infarctus ou sur la perfusion tissulaire dans des modèles d'ischémie périphérique, des études cliniques ont été menées, dès 2001, pour traiter des patients présentant une ischémie cardiaque ou périphérique avec des cellules circulantes, dérivées de la moelle osseuse ou d'autres tissus.

\section{Les différents types de cellules souches adultes}

Cellules souches embryonnaires et adultes

D'un point de vue sémantique, une cellule souche est une cellule indifférenciée, capable de s'autorenouveler et de se différencier en des types cellulaires variés. Il existe plusieurs types de cellules souches:

- des cellules souches multipotentes capables de donner naissance à plusieurs types cellulaires: par exemple les cellules souches hématopoïétiques de la moelle osseuse qui sont à l'origine des cellules sanguines ;

- des cellules souches pluripotentes, issues d'un embryon de 5 à 7 jours, capables de donner naissance à plus de 200 types cellulaires représentatifs de tous les tissus de l'organisme;

- des cellules souches totipotentes embryonnaires capables de donner naissance à tous les types de cellules de l'organisme et les seules capables d'assurer le développement complet d'un individu.

Pour la médecine régénérative, deux principaux types de cellules souches sont à considérer: les cellules souches embryonnaires et les cellules souches adultes. II existe également des cellules souches fœtales dans le sang de cordon ombilical. Enfin, il convient d'ajouter une quatrième catégorie puisqu'a été récemment révélée la possibilité d'obtenir, à partir notamment de fibroblastes cutanés, des cellules souches phénotypiquement semblables aux cellules souches embryonnaires: les cellules souches pluripotentes induites (iPS, induced pluripotent stem cells) [1]. Alors que les cellules souches embryonnaires ou les cellules souches pluripotentes induites possèdent une réelle capacité à se 
différencier en plusieurs types de cellules matures appartenant à des organes de natures distinctes, la plasticité cellulaire de la plupart des cellules souches adultes est moindre et celles-ci expriment probablement un phénotype déjà plus différencié. Enfin, il est très probable que ces cellules souches adultes constituent un assemblage hétérogène de différents types cellulaires : non seulement immatures mais également déjà engagés dans un processus de différenciation. Leur utilisation dans le processus de régénération tissulaire apparaît donc plus limitée. Néanmoins, nous focaliserons cette revue sur les différents types de cellules souches adultes et sur leur efficacité thérapeutique dans l'ischémie tissulaire ou dans le traitement de l'infarctus du myocarde.

\section{Cellules souches adultes utilisées en thérapie cellulaire}

Les cellules souches adultes se répartissent en au moins trois groupes distincts: les cellules souches dérivées de la moelle osseuse, le pool circulant de cellules souches ou progénitrices qui dérivent, au moins en partie, de la moelle osseuse, et les cellules souches résidentes tissulaires.

Les cellules souches dérivées de la moelle osseuse sont les mieux caractérisées et ont été utilisées dans la plupart des essais cliniques. La moelle osseuse contient un assortiment complexe de cellules souches/progénitrices parmi lesquelles les cellules souches hématopoïétiques, les cellules souches mésenchymateuses, une population de cellules définie par leur exclusion du colorant Hoechst et appelée side population ${ }^{1}$, les cellules stromales et les cellules progénitrices multipotentes adultes ${ }^{2}$. Plusieurs études ont étayé le potentiel thérapeutique de l'administration de chacun de ces types cellulaires; certaines ont aussi montré l'intérêt de l'administration des cellules mononucléées totales de la moelle osseuse dans la survie du tissu ischémique [2].

\section{Progéniteurs endothéliaux circulants}

Les cellules progénitrices circulantes représentent la seconde population de cellules progénitrices dont le potentiel thérapeutique a été analysé. Asahara et Isner ont été les premiers à identifier des cellules progénitrices endothéliales (CPE) définies par leur capacité à se différencier en cellules endothéliales, à former des nouveaux capillaires et à initier la revascularisation post-ischémique. Leur capacité de prolifération élevée les distingue des cellules endothéliales matures détachées de la paroi vasculaire [3]. Ces CPE sont caractérisées classiquement par l'expression d'au moins deux marqueurs de cellules souches/progéniteurs hématopoïétiques (CD133 et CD34) et d'un marqueur de cellules endothéliales : le récepteur du VEGF (vascular endothelial growth factor) de type 2 (ou KDR ou flk-1). La sous-population de CPECD133+VEGFR2+ mais dépourvue de l'antigène CD34, représen-

${ }^{1}$ Cette appellation vient de l'analyse au cytomètre de flux de cellules de moelle osseuse ayant été incubées avec le colorant Hoechst 33342 : lorsque l'émission de fluorescence est analysée simultanément à deux longueurs d'onde après excitation du Hoechst dans I'UV, on distingue une population minoritaire se distribuant comme une «queue » expulsant le colorant et donc émettant une faible fluoresence, à la différence de la majorité des cellules. Elle a été nommée side population ou SP.

${ }^{2}$ On désigne sous ce terme une population de cellules très rares, dont l'existence est encore controversée, et qui, in vitro, expriment un potentiel de différenciation très large, y compris dans des lignées non mésodermiques. terait des précurseurs en amont des CPE CD34+CD133+ et exprimerait un potentiel de régénération vasculaire marqué [4]. Cependant, l'identification des CPE reste un problème majeur. II a ainsi été suggéré que la souspopulation $C D 34^{+} C D 45^{-}$, et non celle qui coexprime aussi CD133 et VEGFR2 (CD34+CD133 ${ }^{+}$VEGFR2 $\left.{ }^{+}\right)$, pourrait acquérir un phénotype endothélial [5]. De plus, les cellules myéloides $\mathrm{CD} 14^{+}$peuvent aussi acquérir des caractéristiques de cellules CPE $[6,7]$. Enfin, les cultures de cellules mononucléées de sang périphérique révèlent deux types de CPE : les CPE précoces et les CPE tardives. Les CPE précoces ont des caractéristiques de cellules inflammatoires et induisent la formation des tubes vasculaires indirectement, par une voie paracrine. Les CPE tardives expriment les marqueurs caractéristiques des cellules endothéliales, comme le récepteur du VEGF de type 2 et la $V \varepsilon$-Cadhérine, et se distinguent par leur réel potentiel de différenciation en cellules endothéliales capables de s'incorporer dans les capillaires néoformés. Il est donc probable que ce terme de CPE désigne des cellules circulantes hétérogènes provenant de multiples précurseurs et correspondant à des stades de maturation différents de cellules endothéliales. L'absence de marqueurs définissant spécifiquement la population de CPE productrice de cellules endothéliales fonctionnelles est préjudiciable à une dissection plus précise de ces populations. Il est également plausible que différents types de cellules souches aient un potentiel pro-angiogénique qu'elles expriment via des mécanismes et voies de différenciation divers. Ces deux mécanismes pro-angiogéniques semblent néanmoins acquis: d'une part des cellules progénitrices stimulent la néovascularisation par leur effet paracrine et d'autre part, des CPE « réelles» assurent la formation de néovaisseaux par leur réelle capacité à se différencier en cellules endothéliales.

\section{Autres populations de progéniteurs tissulaires}

Les autres populations de cellules souches/progéniteurs ayant un potentiel thérapeutique dans un contexte ischémique dérivent essentiellement des cellules stromales du tissu adipeux (CPE et cellules souches mésenchymateuses) ou des cellules souches du tissu cardiaque. Le tissu adipeux est une source de différentes sous-populations de cellules souches/progénitrices utiles à la régénération cardiaque et à l'augmentation de la néovascularisation. Des cellules souches mésenchymateuses et des CPE ont été isolées après digestion enzymatique du tissu adipeux et leurs effets bénéfiques ont été démontrés dans des modèles expérimentaux [8]. Plus récemment, plusieurs laboratoires ont isolé des cellules progénitrices dans le tissu cardiaque chez 


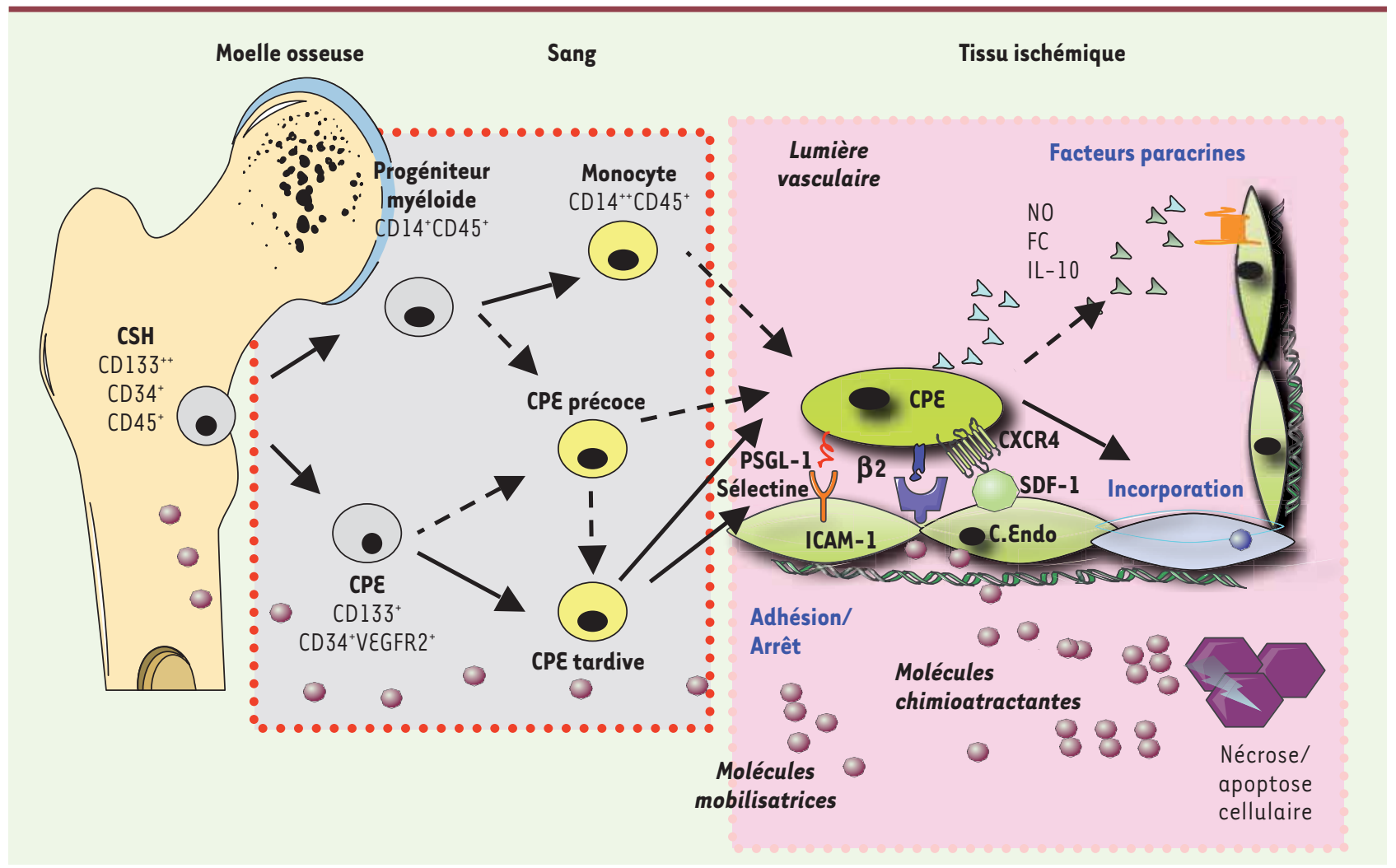

Figure 1. Mécanimes simplifiés d'action des cellules souches de la moelle osseuse dans la réparation vasculaire. Classiquement, il est admis que des signaux provenant du tissu ischémique entraînent la mobilisation de cellules souches de la moelle osseuse vers le sang où elles se différencient en cellules progénitrices endothéliales (CPE). Celles-ci migrent vers la zone lésée et s'arrêtent au niveau de l'endothélium activé par l'ischémie où elles vont exercer leur potentiel pro-angiogénique soit par une action paracrine : libération de monoxyde d'azote (NO), de facteurs de croissance (FC) ou de molécules anti-inflammatoires (comme l'interleukine-10), soit par leur aptitude à se différencier en cellules endothéliales et à s'incorporer dans les néocapillaires. PSGLI: P selectin glycoprotein ligand 1 .

des rongeurs: il s'agit notamment de cellules exprimant c-kit, Sca-1, Islet-l et ou se projetant dans la side population après marquage au Hoechst 33342. Ces cellules souches sont multipotentes in vitro et se différencient in vivo en cardiomyocytes et cellules vasculaires coronaires [9]. Même si ces travaux ont été bien évidemment rapidement remis en question [10], la présence de cellules souches résidant dans le tissu cardiaque représente une avancée majeure dans le domaine de la cardiologie, d'un point de vue biologique mais également sur le plan clinique. Elle permettrait notamment de comprendre l'observation d'un nombre restreint de cardiomyocytes en mitose et la présence de cellules progénitrices vasculaires dans le tissu cardiaque, dont l'origine, médullaire ou endogène cardiaque, est très débattue. Enfin, ces cellules souches cardiaques pourraient servir de base à des thérapies cellulaires développées pour le traitement des pathologies cardiaques [11].

\section{Mécanisme d'action des cellules souches adultes}

En réponse à une ischémie tissulaire, les CPE d'origines médullaire, tissulaire ou périphérique migrent vers le tissu ischémique (Figure l).
Le recrutement des CPE exprimant CXCR4 est stimulé par l'hypoxie et le facteur de transcription HIF-l $\alpha$ qui active l'expression du ligand de CXCR4, la chimiokine SDF-1/CCL12 [12]. Les cellules mononucléées ou les CPE provenant d'animaux $\mathrm{CxCr}^{+/-}$n'ayant qu'un allèle codant pour CXCR4 expriment un nombre diminué de récepteurs CXCR4 et leur capacité à stimuler la reperfusion tissulaire chez des souris immunodéficientes s'en trouve affaiblie [13]. L'hypoxie locale libère également des facteurs solubles chimio-attractants pour les CPE circulantes. Le VEGF est un candidat potentiel dont le taux augmente en conditions hypoxiques et qui induit SDF-1 dans les structures vasculaires et périvasculaires [14]. Les chimiokines et les plaquettes permettent aussi la capture des CPE au niveau des zones de lésions vasculaires $[15,16]$. Une fois qu'elles ont été recrutées, les CPE se fixent sur l'endothélium activé par l'ischémie, grâce à l'action des molécules d'adhésion présentes sur la surface vasculaire comme l'intégrine $\beta 2$ et la sélectine de type L [17-19]. Une fois adhérentes à 


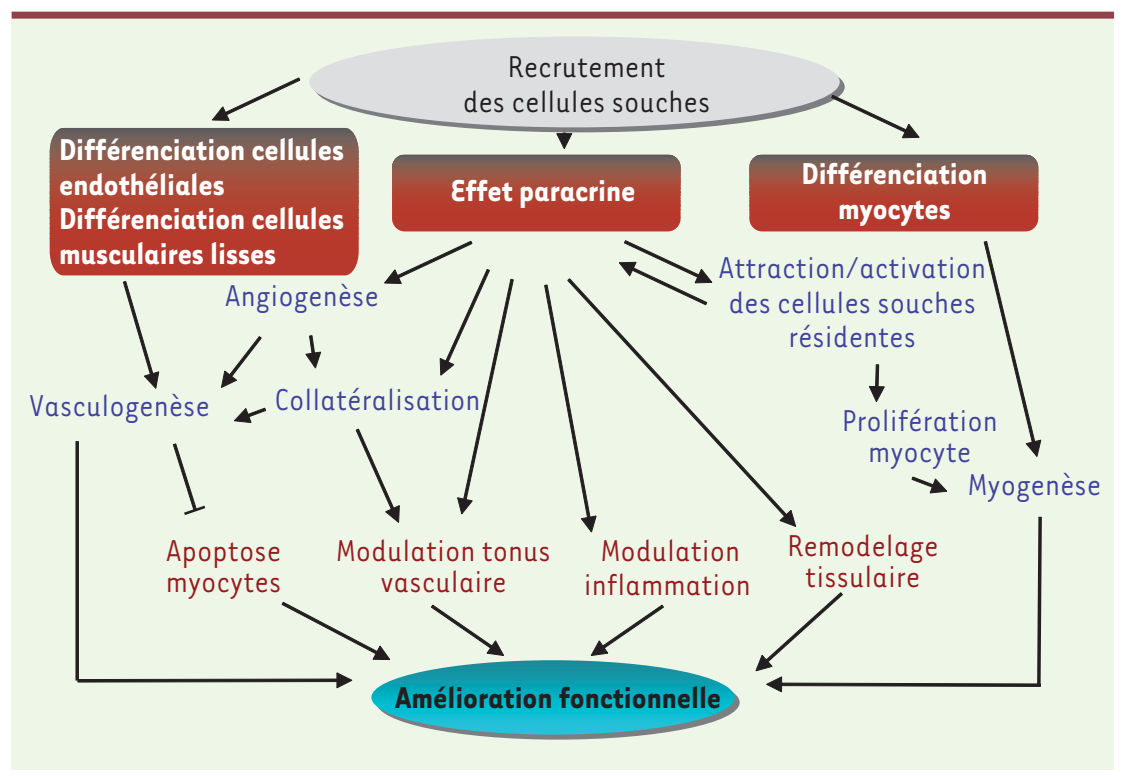

Figure 2. Mécanismes à l'origine de l'efficacité thérapeutique des cellules souches. Ils reposent sur des mécanismes moléculaires et cellulaires complexes: potentiel de différenciation soit en cellules vasculaires ou myocytaires et activité paracrine. II en résulte une modulation quantitative (vasculogenèse et angiogenèse) et qualitative (collatéralisation et inflexion du tonus vasculaire) du compartiment vasculaire mais également myocytaire.

l'endothélium activé par l'ischémie, les CPE exercent leurs effets bénéfiques sur la néogenèse vasculaire par des mécanismes variés (Figure 2).

Selon une première hypothèse, les progéniteurs s'incorporaient dans le site de néovascularisation où ils activent leur différenciation en cellules endothéliales matures [20]. Cependant, la contribution des CPE à la néogenèse vasculaire est très variable selon le modèle expérimental, le type et le sous-type de progéniteurs ou encore la méthode d'isolement des CPE. Certains travaux expérimentaux suggèrent même que ces progéniteurs ne s'incorporent pas dans les néocapillaires mais interviennent surtout par leur rôle de cellules de soutien: les progéniteurs d'origine médullaire s'accumulent autour des vaisseaux collatéraux et se différencient en fibroblastes, leucocytes et péricytes source de facteurs de croissance et de chimiokines [21]. La libération de SDF-1 par les plaquettes favorise la mobilisation et le recrutement des progéniteurs CXCR4 ${ }^{+} V E G F R 1^{+}$autour des structures vasculaires qui, à leur tour, promeuvent le développement de vaisseaux collatéraux [22]. Ainsi, le rôle premier des progéniteurs pourrait être de libérer des facteurs angiogéniques et artériogéniques contribuant ainsi à la régénération vasculaire par des effets paracrines. Par leur capacité à réguler le tonus vasculaire et à induire une vasodilatation via un mécanisme dépendant de la voie eNOS (endothelial nitric oxide synthase), les cellules progénitrices sont également susceptibles de participer à l'amélioration de la perfusion tissulaire dans le territoire ischémié [23]. Enfin, les cellules progénitrices, par leur sécrétion de cytokines anti-inflammatoires comme l'interleukine-10, peuvent améliorer la fonction cardiaque après un infarctus du myocarde [24].

Cependant, les effets paracrines et le potentiel de différenciation en cellules vasculaires ou cardiaques des cellules souches/progéniteurs adultes ne doivent pas être opposés, mais plutôt considérés comme complémentaires. Ainsi, dans un modèle d'ischémie des membres inférieurs et du myocarde chez la souris nude greffée avec des cellules humaines pro-angiogéniques transduites avec un gène « suicide » (séquence du gène $T K$, thymidine kinase, du virus herpes simplex de type 2), l'induction de la mort des cellules progénitrices par le ganciclovir 14 jours après leur injection annule le bénéfice thérapeutique sur la fonction cardiaque. Cela révèle ainsi que l'incorporation physique et la persistance des cellules contribuent à l'effet de ces dernières sur la vascularisation et la fonction cardiaque [25] et conforte l'hypothèse d'une complémentarité des mécanismes paracrines et intrinsèques.

\section{Applications cliniques}

Ces thérapies cellulaires sont actuellement testées dans des essais cliniques incluant de petites cohortes de patients. La moelle osseuse est, pour l'instant, la principale source de cellules souches adultes à la base des thérapies cellulaires pro-angiogéniques en situation autologue. La transition rapide de la démarche expérimentale à l'application clinique a été facilitée par plus de trente ans d'expérience clinique dans le domaine des transplantations de moelle osseuse et par l'absence d'effet délétère de ces transplantations pour la reconstitution du système hématopoïétique. Certes ces cellules médullaires sont un mélange de cellules matures et immatures avec les avantages et inconvénients propres à chacune de ces catégories, mais leur facilité d'obtention permet d'envisager leur utilisation en situation aiguë. Les autres types de cellules souches adultes requièrent une étape d'amplification ex vivo, ce qui impose un délai entre le moment du prélèvement et l'administration des cellules. Une alternative serait de 


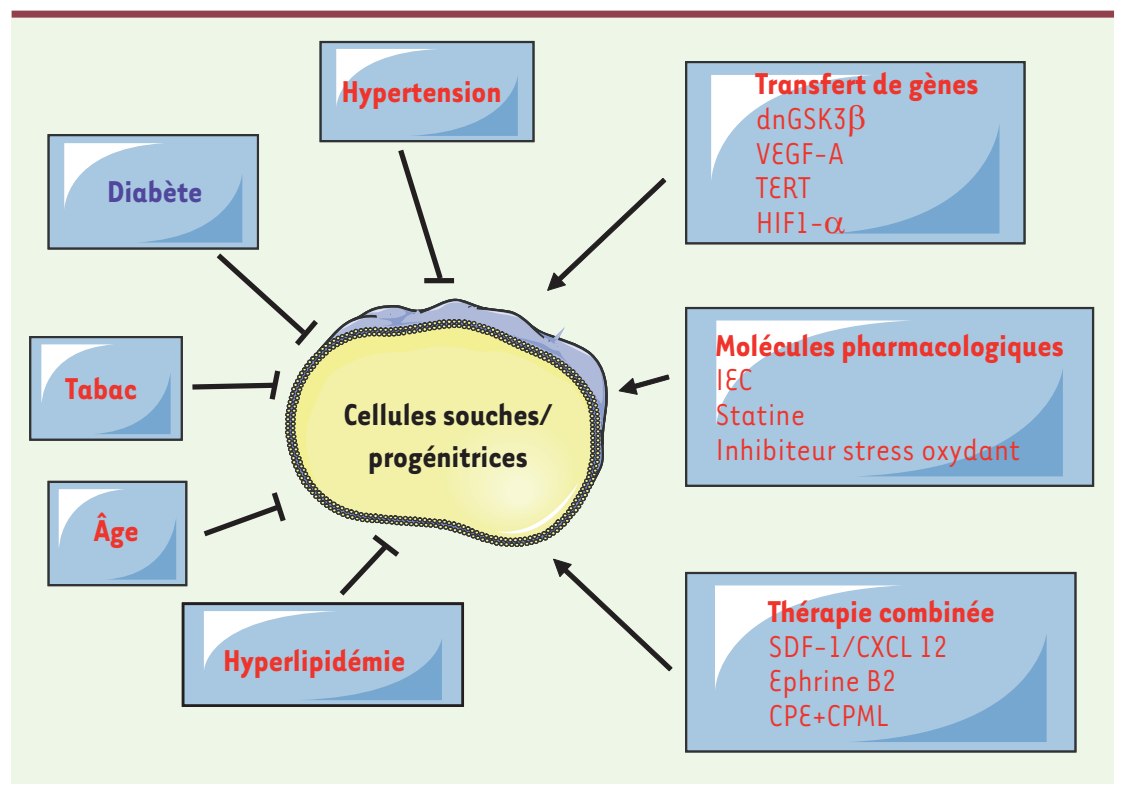

Figure 3. Altération fonctionnelle des cellules souches chez les patients à risque cardiovasculaire et stratégies d'amélioration. La plupart des facteurs de risque cardiovasculaire inhibent la fonction des cellules souches/progénitrices. Dans le cas de la transplantation autologue de cellules souches adultes pour le traitement des patients ischémiques, un certain nombre de stratégies peuvent être envisagées pour restaurer la fonction de ces cellules: transfert de gènes stimulant la prolifération, prétraitement par des molécules pharmacologiques ou encore co-traitements utilisant l'Ephrine B2 ou différents types de cellules progénitrices: dnGSK3 $\beta$, dominant négatif de la protéine kinase GSK3 $\beta$; TERT, télomérase transcriptase inverse ; $I E C$, inhibiteur de l'enzyme de conversion, SDF-1, facteur dérivé du stroma (ligand de CXCR4), CPML, cellules progénitrices musculaires lisses. disposer d'une banque de cellules autologues, une autre de greffer des cellules allogéniques.

L'implantation autologue de cellules mononucléées médullaires dans le membre ischémié de patients souffrant d'une maladie artérielle périphérique ou de thromboangiopathie oblitérante améliore la vascularisation et le processus cicatriciel $[26,27]$. Cependant, un certain nombre de ces patients présentent des effets secondaires, et l'amélioration n'est souvent que transitoire [27]. De même, la transplantation par voie intracoronaire de cellules mononucléées médullaires dans le myocarde de patients ayant une ischémie aiguë augmente la perfusion et la fonction du tissu ischémié [28-30]. Dans cette indication également, certaines études montrent le caractère transitoire de l'amélioration, d'autres une absence d'effet, soulignant ainsi la nécessité de poursuivre l'analyse à la fois expérimentale et clinique de l'efficacité thérapeutique de ces stratégies $[31,32]$.

\section{Quelles stratégies pour optimiser les thérapies cellulaires pro-angiogéniques?}

Des études récentes ont démontré que la plupart des facteurs de risque cardiovasculaire comme le diabète, l'hypertension ou l'âge affectent la fonction des cellules souches adultes [33, 34]. Cette altération des cellules souches du patient susceptibles d'être utilisées pour une transplantation autologue incite à réfléchir à des stratégies visant à restaurer leur activité afin de ne pas compromettre leur efficacité thérapeutique (Figure 3). Elles pourraient être de plusieurs types.

\section{Modifications génétiques}

Ainsi, I'inhibition de la GSK3 3 (glycogen synthase kinase 3) augmente la sécrétion de VEGF et d'interleukine-8, la survie et la prolifération des CPE précoces et la survie et la différenciation des CPE tardives [35]. Le transfert de gènes codant pour le VEGFAl64 ou pour la sous-unité catalytique de la télomérase Tert (telomerase reverse transcriptase) augmente également les activités mitogénique et migratoire et le potentiel thérapeutique des CPE dans un modèle d'ischémie du membre inférieur chez la souris immunodéficiente [36, 37]. Enfin, le transfert du gène codant pour HIF-l $\alpha$ est accompagné d'une augmentation d'expression du VEGF et de son récepteur VEGFR2. II en résulte in vivo une plus grande efficacité des CPE transfectées sur le processus de revascularisation post-ischémique [38].

\section{Associer thérapie cellulaire}

\section{et stratégies pro-angiogéniques}

Des thérapies combinant la transplantation de CPE avec des stratégies pro-angiogéniques pourraient corriger le déficit fonctionnel des CPE chez les patients ischémiques. L'utilisation de molécules pharmacologiques - les statines par exemple - inhibe la sénescence des CPE et induit leur prolifération [39]. Les inhibiteurs de l'enzyme de conversion (IEC) activent la néogenèse vasculaire en stimulant la voie dépendante de la bradykinine [40]. Le traitement de patients coronariens par l'IEC Ramipril augmente le nombre de CPE circulantes et leur activité biologique [41]. Un effet additif a même été observé entre l'administration intraveineuse de CPE et celle d'un IEC sur la fonction ventriculaire de rats immunodéficients chez lesquels un infarctus du myocarde avait été induit [42]. Enfin, l'utilisation d'inhibiteurs du stress oxydant restaure la capacité de cellules mononucléées médullaires de souris diabétiques ou de rats hypertendus à se différencier en cellules endothéliales et à démarrer un processus de néogenèse vasculaire dans la zone ischémiée $[43,44]$. 


\section{Associer thérapies génique et cellulaire}

Cette combinaison présente un intérêt. Ainsi, l'injection locale de SDF-l dans la patte ischémique de souris immunodéficientes associée à l'administration intraveineuse de CPE humaines augmente l'accumulation tissulaire de CPE dans la zone lésée et leur efficacité thérapeutique [45]. Un prétraitement par l'éphrine B2 améliore également la capacité de migration des CPE vers la zone ischémique et ainsi leur potentiel thérapeutique dans un modèle d'ischémie du membre inférieur [46]. De même, l'administration simultanée de CPE et de progéniteurs de cellules musculaires lisses est bénéfique car la libération de l'angiopoïétine-2 par ces derniers active la survie des CPE dans le territoire ischémique et ainsi facilite la néovascularisation [47].

\section{Conclusion}

Il apparaît donc clairement que l'administration de cellules souches ou progénitrices adultes pour la régénération tissulaire est encore loin d'être une stratégie de routine en pratique clinique. Une attention toute particulière doit être apportée aux types de cellules souches utilisées, à leur origine, leur pureté, leur capacité de différenciation et à leur potentiel thérapeutique, en particulier si elles sont utilisées pour une transplantation autologue chez des patients ischémiques déjà porteurs de facteurs de risque cardiovasculaire importants. L'avenir de la thérapie cellulaire pro-angiogénique réside peut-être dans la transplantation allogénique de cellules souches provenant de sang de cordon ombilical. Mais se pose le problème de la compatibilité immunologique et du développement des banques de sang de cordon, notamment en France. Enfin, même si l'utilisation de cellules souches embryonnaires pourrait, en partie, résoudre les difficultés fonctionnelles inhérentes aux cellules souches adultes, leur potentiel thérapeutique doit être évalué au regard des risques inhérents à leur utilisation dans les tissus pathologiques chez les patients ischémiques [48]. $\diamond$

\section{SUMMARY}

Proangiogenic cell-based therapy

for treatment of ischemic diseases

The application of endothelial progenitor cells (EPC) cell-based therapy for regenerative medicine constitutes a promising therapeutic avenue for the treatment of cardiovascular diseases. Based on experimental studies demonstrating that bone marrow-, blood- or tissue-derived stem/progenitor cells improve the functional recovery after ischemia, clinical trials were initiated to address this new therapeutic concept. Although autolougous cell therapy was shown to improve perfusion and function of ischemic tissues, a number of issues remain to be adressed. The nature of the mobilizing, migratory and homing signals, and the mechanisms of action need to be identified and further defined. In addition, strategies to enhance homing, survival and therapeutic potential of EPC need to be developped to improve therapeutic effect and counteract EPC dysfunction in aged patients with cardiovascular risk factors. The present review article will discuss the mechanisms of action of different types of adult stem cells and several approaches to improve their therapeutic efficiency. $\diamond$

\section{RéFÉRENCES}

1. Takahashi K, Yamanaka S. Induction of pluripotent stem cells from mouse embryonic and adult fibroblast cultures by defined factors. Cell $2006 ; 126: 663-76$.

2. Dimmeler S, Burchfield J, Zeiher AM. Cell-based therapy of myocardial infarction. Arterioscler Thromb Vasc Biol 2008 ; 28 : 208-16.

3. Rafii S, Lyden D. Therapeutic stem and progenitor cell transplantation for organ vascularization and regeneration. Nat Med $2003 ; 9: 702-12$.

4. Friedrich EB, Walenta K, Scharlau J, et al. CD34-/CD133+/VEGFR-2+ endothelial progenitor cell subpopulation with potent vasoregenerative capacities. Circ Res 2006 ; 98 : e20-25.

5. Case J, Mead LE, Bessler WK, et al. Human CD34+AC133+VEGFR-2+ cells are not endothelial progenitor cells but distinct, primitive hematopoietic progenitors. Exp Hematol 2007 ; 35 : 1109-18.

6. Urbich C, Heeschen C, Aicher A, et al. Relevance of monocytic features for neovascularization capacity of circulating endothelial progenitor cells. Circulation $2003 ; 108: 2511-6$.

7. Rehman J, Li J, Orschell CM, et al. Peripheral blood endothelial progenitor cells are derived from monocyte/macrophages and secrete angiogenic growth factors. Circulation $2003 ; 107$ : 1164-9.

8. Planat-Benard V, Silvestre JS, Cousin B, et al. Plasticity of human adipose lineage cells toward endothelial cells: physiological and therapeutic perspectives. Circulation $2004 ; 109: 656-63$.

9. Beltrami AP, Barlucchi L, Torella D, et al. Adult cardiac stem cells are multipotent and support myocardial regeneration. Cell 2003; 114 : 763-76.

10. Laflamme MA, Murry C $\varepsilon$. Regenerating the heart. Nat Biotechnol 2005 ; $23: 845-56$.

11. Dawn B, Stein AB, Urbanek K, et al. Cardiac stem cells delivered intravascularly traverse the vessel barrier, regenerate infarcted myocardium, and improve cardiac function. Proc Natl Acad Sci USA 2005 ; $102: 3766-71$.

12. Ceradini DJ, Gurtner GC. Homing to hypoxia: HIF-l as a mediator of progenitor cell recruitment to injured tissue. Trends Cardiovasc Med $2005 ; 15: 57-63$.

13. Walter DH, Haendeler J, Reinhold J, et al. Impaired CXCR4 signaling contributes to the reduced neovascularization capacity of endothelial progenitor cells from patients with coronary artery disease. Circ Res 2005; 97 : 1142-51.

14. Grunewald M, Avraham I, Dor Y, et al. VEGF-induced adult neovascularization: recruitment, retention, and role of accessory cells. Cell 2006; $124: 175-89$.

15. Waeckel L, Mallat Z, Potteaux S, et al. Impairment in postischemic neovascularization in mice lacking the $\mathrm{CXC}$ chemokine receptor 3 . Circ Res $2005 ; 96: 576-82$.

16. Langer $H$, May AE, Daub K, et al. Adherent platelets recruit and induce differentiation of murine embryonic endothelial progenitor cells to mature endothelial cells in vitro. Circ Res $2006 ; 98:$ e2-10.

17. Chavakis $\varepsilon$, Aicher A, Heeschen C, et al. Role of beta2-integrins for homing and neovascularization capacity of endothelial progenitor cells. J Exp Med $2005 ; 201: 63-72$

18. Biancone L, Cantaluppi V, Duo D, et al. Role of L-selectin in the vascular homing of peripheral blood-derived endothelial progenitor cells. J Immunol $2004 ; 173$ : 5268-74.

19. Yoon CH, Hur J, Oh ly, et al. Intercellular adhesion molecule- 1 is upregulated in ischemic muscle, which mediates trafficking of endothelial progenitor cells. Arterioscler Thromb Vasc Biol 2006; $26: 1066-72$.

20. Takahashi T, Kalka C, Masuda H, et al. Ischemia- and cytokine-induced mobilization of bone marrow-derived endothelial progenitor cells for neovascularization. Nat Med $1999 ; 5: 434-8$.

21. Ziegelhoeffer T, Fernandez B, Kostin S, et al. Bone marrow-derived cells do not incorporate into the adult growing vasculature. Circ Res 2004 ; $94: 230-8$.

22. Jin DK, Shido K, Kopp HG, et al. Cytokine-mediated deployment of SDF-1 induces revascularization through recruitment of CXCR4+ hemangiocytes. Nat Med $2006 ; 12: 557-67$.

23. You D, Waeckel L, Ebrahimian TG, et al. Increase in vascular permeability and vasodilation are critical for proangiogenic effects of stem cell therapy. Circulation 2006; $114: 328-38$.

24. Burchfield JS, Iwasaki M, Koyanagi M, et al. Interleukin-10 from transplanted bone marrow mononuclear cells contributes to cardiac protection after myocardial infarction. Circ Res 2008 ; 103 : 203-11. 
25. Ziebart T, Yoon CH, Trepels T, et al. Sustained persistence of transplanted proangiogenic cells contributes to neovascularization and cardiac function after ischemia. Circ Res 2008 ; $103: 1327-34$.

26. Tateishi-Yuyama $\varepsilon$, Matsubara H, Murohara T, et al. Therapeutic angiogenesis for patients with limb ischaemia by autologous transplantation of bone-marrow cells: a pilot study and a randomised controlled trial. Lancet $2002 ; 360: 427-35$.

27. Miyamoto K, Nishigami K, Nagaya N, et al. Unblinded pilot study of autologous transplantation of bone marrow mononuclear cells in patients with thromboangiitis obliterans. Circulation 2006 ; $114: 2679-84$.

28. Schachinger V, Assmus B, Britten MB, et al. Transplantation of progenitor cells and regeneration enhancement in acute myocardial infarction: final one-year results of the TOPCARE-AMI Trial. J Am Coll Cardiol 2004 ; $44: 1690-9$.

29. Assmus B, Honold J, Schachinger V, et al. Transcoronary transplantation of progenitor cells after myocardial infarction. N Engl/ Med $2006 ; 355$ : 1222-32.

30. Schachinger $V$, Erbs $S$, Elsasser $A$, et al. Intracoronary bone marrow-derived progenitor cells in acute myocardial infarction. $N$ Engl J Med 2006 ; 355 : 1210-21.

31. Lunde K, Solheim S, Aakhus $S$, et al. Intracoronary injection of mononuclear bone marrow cells in acute myocardial infarction. $N$ Engl J Med 2006 ; 355 : 1199-209.

32. Meyer GP, Wollert KC, Lotz J, et al. Intracoronary bone marrow cell transfer after myocardia infarction: eighteen months' follow-up data from the randomized, controlled BO0ST (Bone marrow transfer to enhance ST-elevation infarct regeneration) trial. Circulation 2006; $113: 1287-94$.

33. You D, Cochain C, Loinard C, et al. Hypertension impairs postnatal vasculogenesis: role of antihypertensive agents. Hypertension 2008 ; 51 : 1537-44.

34. Tamarat R, Silvestre JS, Le Ricousse-Roussanne $S$, et al. Impairment in ischemia-induced neovascularization in diabetes: bone marrow mononuclear cell dysfunction and therapeutic potential of placenta growth factor treatment. Am J Pathol $2004 ; 164: 457-66$.

35. Choi JH, Hur J, Yoon CH, et al. Augmentation of therapeutic angiogenesis using genetically modified human endothelial progenitor cells with altered glycogen synthase kinase-3beta activity. J Biol Chem $2004 ; 279: 49430-8$.

36. Iwaguro H, Yamaguchi J, Kalka C, et al. Endothelial progenitor cell vascular endothelial growth factor gene transfer for vascular regeneration. Circulation $2002 ; 105: 732-8$.

37. Murasawa S, Llevadot J, Silver M, et al. Constitutive human telomerase reverse transcriptase expression enhances regenerative properties of endothelial progenitor cells. Circulation $2002 ; 106: 1133-9$.

38. Jiang $M$, Wang $B$, Wang $C$, et al. Angiogenesis by transplantation of HIF-l alpha modified EPCS into ischemic limbs. J Cell Biochem 2008; 103: 321-34.
39. Assmus B, Urbich C, Aicher A, et al. HMG-CoA reductase inhibitors reduce senescence and increase proliferation of endothelial progenitor cells via regulation of cell cycle regulatory genes. Circ Res $2003 ; 92$ : 1049-55.

40. Silvestre JS, Bergaya S, Tamarat R, et al. Proangiogenic effect of angiotensin-converting enzyme inhibition is mediated by the bradykinin $B(2)$ receptor pathway. Circ Res $2001 ; 89: 678-83$.

41. Min TQ, Zhu CJ, Xiang WX, et al. Improvement in endothelial progenitor cells from peripheral blood by ramipril therapy in patients with stable coronary artery disease. Cardiovasc Drugs Ther $2004 ; 18$ : 203-9.

42. Boyle AJ, Schuster M, Witkowski $P$, et al. Additive effects of endothelia progenitor cells combined with ACE inhibition and beta-blockade on left ventricular function following acute myocardial infarction. J Renin Angiotensin Aldosterone Syst $2005 ; 6$ : 33-7.

43. Ebrahimian TG, Heymes C, You D, et al. NADPH oxidase-derived overproduction of reactive oxygen species impairs postischemic neovascularization in mice with type 1 diabetes. Am J Pathol 2006 ; $169: 719-28$

44. You D, Cochain C, Loinard C, et al. Combination of the angiotensin converting enzyme inhibitor Perindopril and the diuretic Indapamide activate post-natal vasculogenesis in Spontaneaously Hypertensive Rats. J Pharmacol Exp Ther $2008 ; 325: 766-73$.

45. Yamaguchi J, Kusano KF, Masuo 0, et al. Stromal cell-derived factor-1 effects on ex vivo expanded endothelial progenitor cell recruitment for ischemic neovascularization. Circulation $2003 ; 107$ : 1322-8.

46. Foubert P, Silvestre JS, Souttou B, et al. PSGL-1-mediated activation of $\varepsilon p h B 4$ increases the proangiogenic potential of endothelial progenitor cells. J Clin Invest $2007 ; 117: 1527-37$.

47. Foubert $P$, Matrone $G$, Souttou $B$, et al. Coadministration of endothelial and smooth muscle progenitor cells enhances the efficiency of proangiogenic cell-based therapy. Circ Res 2008; 103 : 751-60.

48. Peters BA, Diaz LA, Polyak K, et al. Contribution of bone marrow-derived endothelial cells to human tumor vasculature. Nat Med 2005; 11:261-2.

\section{TIRÉS À PART}

J.S. Silvestre

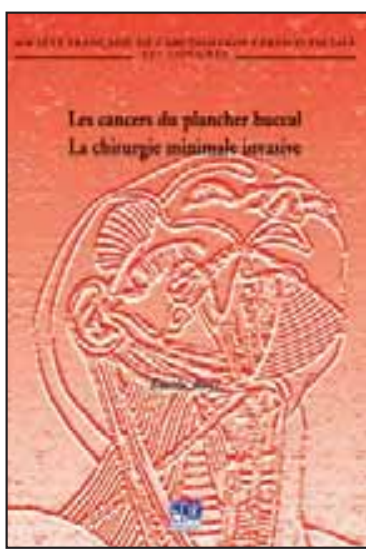

ISBN : 978-2-8425-4127-9 212 pages

\section{Bon de commande}

À retourner à EDK, 2, rue Troyon - 92316 Sèvres Cedex

Tél. : 0155641393 - Fax : 0155641394 - E-mail : edk@edk.fr

NOM :

Prénom

Adresse :

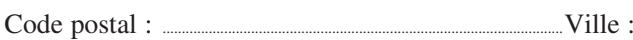

Pays :

Fonction :

Je souhaite recevoir l'ouvrage Les cancers du plancher buccal : $35 €+3 €$ de port $=\mathbf{3 8} €$ TTC

en ................. exemplaire, soit un total de ..................................... €

$\square$ Par chèque, à l'ordre de $\mathbf{E} \mathbf{D} \mathbf{K}$

$\square$ Par carte bancaire : $\square$ Visa $\square$ Eurocard/Mastercard

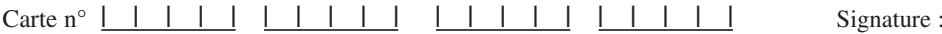

Date d'expiration: $\quad \underline{1}|\underline{1}|$

$\mathrm{N}^{\circ}$ de contrôle au dos de la carte : $\quad \underline{\mathrm{I} \quad \mathrm{I}}$ 


\section{Ateliers de formation 2010}

Renseignements et inscriptions :

Ateliers de formation Inserm

101 , rue de Tolbiac

75654 Paris Cedex 13

Tél. : 33 (0)1 44236204 - Fax : 33 (0)1 44236293

ateliers@inserm.fr

ww w.rh.inserm. fr

\section{Atelier de formation $n^{\circ} 202$}

\section{Recherche in silico de sondes pharmacologiques et candidats médicaments : succès et défis}

Organisateurs : M aria M iteva (M TI, Inserm UM R-S 973 - Université Paris Diderot), Véronique Stoven (Inserm U900, M ines Paris Tech, Institut Curie), Bruno Villoutreix (M TI, Inserm UM R-S 973 - Université Paris Diderot).

\section{Phase I $\bullet$ Le point sur... 23-25 mars 2010 - Saint-Raphaël}

Objectifs - Les méthodes in silico jouent un rôle important dans la recherche biomédicale actuelle. Les approches in silico ont été utilisées pendant de nombreuses années pour faciliter la recherche de molécules actives, mais un rapport récent montre que ces outils peuvent aussi aider à la découverte de médicaments. De nombreux exemples de molécules touches (" hits ») inhibant des enzymes ou bloquant des interactions macromoléculaires identifiées par criblage virtuel ont été publiés. L'objectif de l'atelier est d'introduire les méthodes in silico "ligand-based » et "structure-based » actuellement utilisées et de présenter des exemples d'applications de ces méthodes à la découverte de touches innovantes. Ainsi, cet atelier présentera à la fois les aspects théoriques des méthodes existantes et des nouvelles approches et des exemples d'applications sur des cibles thérapeutiques pertinentes. II montrera aux participants comment de telles méthodes in silico combinées aux approches expérimentales peuvent aider à la découverte rapide et à coûts réduits de nouvelles molécules innovantes. Cet atelier va permettre d'adopter un langage commun (biologie-chémoinformatique) et facilitera la mise en place de collaborations entre les chémoinformaticiens et les biologistes.

Public • Une large audience scientifique académique ou industrielle (chercheurs, ingénieurs, post-doctorants et doctorants) intéressée par la découverte de molécules bioactives «drug-like » ou de sondes chimiques : bio/ chemo-informaticiens; chercheurs biologistes et chimistes : le but est de présenter les méthodologies in silico qui a ideront à réaliser (ou initier) des projets à l'interface chimie-biologie axés sur la conception de nouvelles molécules touches. Les conférences seront données en anglais.

\section{Nombre maximum de participants : 80}

Programme - 1. Criblage virtuel basé sur la structure de récepteur : les méthodes existantes et les nouveaux concepts. 2. Recherche de molécules touches par des méthodes basées sur la structure de ligands connus.

3. "Success stories » : couplage des approches in silico-in vitro pour la découverte de nouvelles molécules d'intérêt pharmacologique.

4. Valorisation: transfert de technologies et brevets; plateformes de criblage en France.

La $4^{\mathbf{e}}$ journée annuelle nationale en chémoinformatique organisée par la Société Française de Chémoinformatique se tiendra le 26 mars 2010 à Saint-Raphaël (informations supplémentaires sur http:/ / ww w. chemoinformatique.fr/ modules/ smartsection/ item. php? itemid $=32$ ).

\section{Phase II • Maîtrise technique}

\section{8-10 novembre 2010 • MTI, Inserm UMR-S 973 - Université Paris Diderot}

Programme - Travaux pratiques sur ordinateur, visant à l'introduction des outils permettant: $A$. Préparation de chimiothèques des petits composés (pour tous les participants) : prédiction de certaines propriétés ADM E-Tox ; génération et optimisation de structures 3D ; B. C riblage virtuel basé sur la structure du récepteur ; $C$. C riblage virtuel basé sur des ligand connus. Chaque participant choisira entre le stage B ou C selon ses projets.

Sélection • 15 participants sélectionnés parmi les participants de la phase I.

Avec la participation de - Ruben Abagyan (La Jolla, USA), Andreas Bender (Leiden, The N etherlands), John Hickmann (Paris), Denise Hirsch (Paris), Richard Jackson (Leeds, UK), Gerard Kleywegt (Cambridge, UK), Bernard M aigret (N ancy), M aria M iteva (Paris), Xavier Morelli (Marseille), Stefano Moro (Padova, Italy), O livier Sperandio (Paris), Jean-Philippe Vert (Paris), Michel Vidal (Paris), Michael W iese (Bonn, Germany).

Date limite d'inscription : 25 janvier 2010 